\title{
Combined treatment with PI3K inhibitor BKM120 and PARP inhibitor olaparib is effective in inhibiting the gastric cancer cells with ARID1A deficiency
}

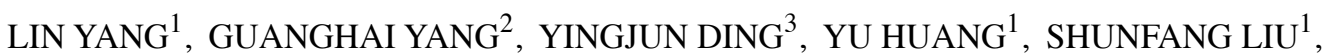 \\ LEI ZHOU ${ }^{1}$, WENJIE WEI ${ }^{1}$, JING WANG ${ }^{1}$ and GUANGYUAN HU ${ }^{1}$
}

${ }^{1}$ Department of Oncology, Tongji Hospital, ${ }^{2}$ Department of Thoracic Surgery, Union Hospital, Tongji Medical College,
Huazhong University of Science and Technology, Wuhan, Hubei 430030, P.R. China;
${ }^{3}$ Department of Physiology, University of Oklahoma Health Sciences Center, Oklahoma City, OK 73104, USA

Received November 17, 2017; Accepted April 26, 2018

DOI: 10.3892/or.2018.6445

\begin{abstract}
Dual blockade of phosphoinositide 3-kinase (PI3K) and poly(ADP-ribose) polymerase (PARP) has been revealed to be an effective treatment strategy for breast, ovarian and prostate cancer. However, the efficacy of this combination for the treatment of gastric cancer, and potential predictive therapeutic biomarkers remain unclear. Recent evidence suggests that the deficiency of AT-rich interactive domain containing protein 1A (ARIDIA), which is a crucial chromatin remodeling gene, sensitizes tumor cells to PI3K and PARP inhibitors. Herein, we evaluated the therapeutic role of the combined treatment of PI3K inhibitor BKM120 and PARP inhibitor olaparib on gastric cancer cells, and explored ARID1A as a predictive biomarker. The results demonstrated that combined treatment with PI3K and PARP inhibitors effectively inhibited proliferation detected by MTS and clonogenic assay, invasion and migration by Transwell assay, of gastric cancer cells with ARID1A deficiency. Mechanistically, dual blockade of PI3K and PARP in ARID1A-depleted gastric cancer cells significantly increased apoptosis detected by flow cytometry, and induced DNA damage by immunofluorescent staining. Taken together, these data suggest that the combined treatment with PI3K inhibitor BKM120 and PARP inhibitor olaparib may be a promising therapeutic regimen for the treatment of gastric
\end{abstract}

Correspondence to: Professor Guangyuan $\mathrm{Hu}$, Department of Oncology, Tongji Hospital, Tongji Medical College, Huazhong University of Science and Technology, 1095 Jiefang Avenue, Wuhan, Hubei 430030, P.R. China

E-mail: h.g.y.121@163.com

Abbreviations: ARID1A, AT-rich interactive domain containing protein 1A; PARP, poly(ADP-ribose) polymerase; PI3K, phosphoinositide 3-kinase; $\mathrm{p}-\mathrm{H} 3$, phosphorylated histone H3 on Ser10

Key words: ARID1A, gastric cancer, PI3K, PARP cancer, and ARID1A deficiency could serve as a potential predictive therapeutic biomarker.

\section{Introduction}

Gastric cancer ranks as the fourth most common cancer, and remains the second leading cause of cancer-related death worldwide (1). High incidence areas include Asia, Central and Eastern Europe and South America (2). Despite the steadily declining incidence, the clinical outcome of gastric cancer has improved modestly in recent years. In addition, in 2013, more than 841,000 deaths were attributed to gastric cancer (3). The overall 5-year survival rate is still less than $25 \%$ (4). One main leading cause is the poor response of gastric cancer to currently available treatments (5). Thus, currently, targeted drugs are being explored extensively, which may afford new therapeutic avenues for gastric cancer.

Poly(ADP-ribose) polymerase (PARP) inhibitors are a type of promising targeted drugs, which have been approved by the FDA to be used for the treatment of breast and ovarian cancer patients with mutations of the BRCA1 or BRCA2 genes (6). The PARP inhibitor is designed to target cancers with impaired DNA damage repair abilities, such as mutations of BRCA1/2, which is called 'synthetic lethality' $(7,8)$. However, several other molecular biomarkers have also been revealed to predict the sensitivity to PARP inhibitors, including CDK depletion (9), RAD51C deficiency (10), ATM deficiency (11) and AT-rich interactive domain containing protein 1A (ARID1A) deficiency (12). Nonetheless, in gastric cancer, the most recent clinical trial has reported that olaparib, an oral PARP inhibitor, in combination with paclitaxel did not significantly improve the overall survival in the overall or ATM-negative population of Asian patients (13). Thus, there is an urgent need to identify new drug combinations, and new predictive biomarkers for the treatment of gastric cancer.

Notably, phosphoinositide 3-kinase (PI3K) inhibition has been revealed to impair BRCA1/2 expression, render tumor cells more deficient in HR repair and sensitize breast cancer to PARP inhibition (14). The PI3K signaling pathway plays a critical role in regulating various cellular processes, including 
proliferation, growth, apoptosis and cell metabolism $(15,16)$, and has been demonstrated to be an attractive target for the treatment of various types of cancers. BKM120, a selective pan-class I PI3K inhibitor, has been reported to be effective in gastric cancer (17). In fact, the combined treatment with PI3K inhibitor BKM120 and PARP inhibitor olaparib has been demonstrated to be effective for breast $(14,18)$, prostate (19) and ovarian cancer $(20,21)$. However, the efficacy of the combination in gastric cancer remains unclear, and potential predictive biomarkers must be explored.

Recent evidence indicates that ARID1A deficiency sensitizes tumor cells to PARP and PI3K inhibitors $(12,22)$. $A R I D I A$ is a novel chromatin remodeling gene, which has been identified to be frequently mutated in a broad range of cancers (23-25). The incidence of mutations of ARIDIA in gastric cancer varies from 8 to $27 \%$ (26-28). ARIDIA encodes the protein BRG1-associated factor 250a (BAF250a), which is a crucial non-catalytic subunit of human switch/sucrose nonfermentable (SWI/SNF) complex (29). The SWI/SNF complex plays an important role in transcription, DNA replication and DNA damage repair $(12,30,31)$. Mutations of ARIDIA in tumors usually cause downregulated protein expression (32) and ARID1A is suggested as a bona fide tumor suppressor (33). ARID1A depletion has been reported to promote gastric cancer cell growth in vitro, xenograft tumor growth in vivo (22), and cell migration and invasion (34), which indicate that ARID1A is a potential target for gastric cancer.

Therefore, in the present study, we investigated the therapeutic role of the combined treatment of PI3K inhibitor BKM120 and PARP inhibitor olaparib on gastric cancer cells, and explored ARID1A deficiency as a potential predictive biomarker of therapeutic efficacy.

\section{Materials and methods}

Cell lines and reagents. Human gastric cancer cell lines AGS and SNU-1 were purchased from the Cell Bank of the Shanghai Institutes for Biological Sciences (Shanghai, China). Cells were grown in RPMI-1640 medium (HyClone Laboratories; GE Healthcare, Chicago, IL, USA) supplemented with $10 \%$ fetal bovine serum (FBS; Gibco; Thermo Fisher Scientific, Inc., Waltham, MA, USA). Cells were cultured in a humidified incubator containing $5 \%$ carbon dioxide $\left(\mathrm{CO}_{2}\right)$ at $37^{\circ} \mathrm{C}$. BKM120 and olaparib were obtained from Selleck Chemicals (Houston, TX, USA) and dissolved in dimethyl sulfoxide (DMSO).

Short hairpin RNA (shRNA)-mediated ARIDIA knockdown. Lentivirus-ARID1A-RNAi vector and the corresponding empty vector were purchased from Shanghai GeneChem, Co., Ltd. (Shanghai, China). We designed two independent shRNA constructs and subcloned them into the lentivirus vectors (sequence: shARID1A \#1, 5'-GCCTGATCTATCTGGTTCA AT-3'; shARID1A\#2,5'-CCTCTCTTATACACAGCAGAT-3'). The constructed plasmid was confirmed by DNA sequencing. Then, the lentiviral vector and packaging mix with Lipofectamine 2000 (Invitrogen; Thermo Fisher Scientific, Inc.) were transfected into 293FT cells. The supernatant containing the lentivirus was collected $48 \mathrm{~h}$ later, and was purified and supplemented with $8 \mu \mathrm{g} / \mathrm{ml}$ Polybrene (Santa Cruz
Biotechnology, Inc., Santa Cruz, CA, US). The virus solution was used to infect the target cells. Another $72 \mathrm{~h}$ later, $2 \mu \mathrm{g} / \mathrm{ml}$ of puromycin (Sangon Biotech, Shanghai, China) was added for 1 week to select the stably transfected cells. Then, western blot analysis was used to evaluate the protein expression level.

MTS assay. Cells were seeded into a 96-well culture plate. On the next day, the indicated drugs were used to treat the cells. Seventy-two hours later, the cells were washed and treated with $20 \mu \mathrm{l}$ MTS reagent (Promega, Madison, WI, USA) per well for $2 \mathrm{~h}$. Cell viability was detected by a microplate reader at a wavelength of $490 \mathrm{~nm}$. The half-maximal inhibitory concentration $\left(\mathrm{IC}_{50}\right)$ was analyzed using GraphPad Prism 6.0 (GraphPad Software, Inc., San Diego, CA, USA). The combination index (CI) was calculated by Chou-Talalay method (35). $\mathrm{CI}<1$ indicates synergism, $\mathrm{CI}>1$ indicates antagonism, and $\mathrm{CI}=1$ indicates additive interactions.

Clonogenic assay. Cells were planted into 6-well culture plates. On the next day, the cells were treated with the indicated drugs for 3 days and cultured for 2 weeks. Then, the clones were fixed with $4 \%$ polymerised formaldehyde, and were stained with $0.01 \%$ crystal violet (Sangon Biotech). The number of clones were counted.

Small interfering RNA (siRNA)-mediated ARIDIA knockdown. Cells were planted into a $6-\mathrm{cm}$ cell culture dish, at a density of $30 \%$. On the next day, the cells were transfected using ARIDIA or non-target siRNAs (Shanghai GeneChem) with Oligofectamine transfection reagent (Invitrogen; Thermo Fisher Scientific, Inc.) based on the manufacturer's protocol. The siRNA sequences were as follows (34): ARIDIA, sense, 5'-GCCCUAACAUGGCCAAUAUTT-3' and antisense, 5'-AU AUUGGCCAUGUUAGGGCTT-3'; non-target control: sense, 5'-UUCUCCGAACGUGUCACGUTT-3' and antisense, 5'-AC GUGACACGUUCGGAGAATT-3'.

Apoptosis assay. Cells were seeded into 6-well culture plates at an $\sim 40 \%$ density. On the next day, the cells were exposed to the indicating drugs. After $24 \mathrm{~h}$, the cells were collected and resuspended in binding buffer supplemented with Annexin V-PE. Then, propidium iodide (PI) was added, and the Annexin V/PI apoptosis kit (Invitrogen; Thermo Fisher Scientific, Inc.) was used. The rate of apoptosis was detected by flow cytometric analysis (FACScan; Beckman Coulter, Inc., Brea, CA, USA). In the figures demonstrating the results, the percentage of cells in the upper right (including necrotic or late apoptotic cells) and lower right corners (including early apoptotic cells) were added to obtain the percentage of apoptosis.

Western blot analysis. Cells were harvested and lysed in urine buffer supplemented with $1 \%$ phosphorylation inhibitors and $1 \%$ protease (Roche Diagnostics, Indianapolis, IN, USA). Total protein $(30 \mu \mathrm{g})$ for each sample was loaded to SDS-PAGE gel. After gel electrophoresis, the protein was transferred to polyvinylidene fluoride membranes (EMD Millipore, Billerica, MA, USA). Then, the membranes were blocked using 5\% non-fat dried milk for $1 \mathrm{~h}$, and washed in TBS with $0.1 \%$ Tween-20 for 3 times at room temperature. Then, the membranes were incubated with 
primary antibodies overnight at $4^{\circ} \mathrm{C}$. The membranes were washed for three times, and then incubated with horseradish peroxidise-conjugated goat anti-mouse or goat anti-rabbit secondary antibodies (1:5,000; cat. nos. 31430 and 31460; Invitrogen; Thermo Fisher Scientific, Inc.) for $1 \mathrm{~h}$. After washing for another 3 times, the expression of the target proteins were visualized using an ECL detection kit (Thermo Fisher Scientific, Inc.). The following were the antibodies used in this study: ARID1A (1:500; cat. no. A301-040A; Bethyl Laboratories, Montgomery, TX, USA); BRCA1, BRCA2 and cleaved-PARP (1:500; cat. nos. 9010, 9012 and 9185; Cell Signaling Technology, Inc., Danvers, MA, USA); AKT, p-AKT (Ser473) (1:500; cat. nos. ab28422 and ab81283; Abcam, Cambridge, MA, USA); H2AX, $\gamma \mathrm{H} 2 \mathrm{AX}$ antibodies (1:1,000; cat. nos. DR1016 and 05-636; EMD Millipore; Merck KGaA, Darmstadt, Germany), N-cadherin, E-cadherin, $\beta$-catenin, ZEB1 and vimentin (1:500; cat. no. 9782; Cell Signaling Technology, Inc.) and $\beta$-actin antibody $(1: 2,000$; cat. no. A5441; Sigma-Aldrich; Merck KGaA, Darmstadt, Germany).

Immunofluorescent staining. Cells on coverslips were fixed using $3 \%$ paraformaldehyde for $20 \mathrm{~min}$ at room temperature, and then were permeabilized with phosphate-buffered saline (PBS) containing $0.5 \%$ Triton X-100 for $5 \mathrm{~min}$. PBS containing $5 \%$ goat serum was used to block the coverslips for $30 \mathrm{~min}$. Then, the coverslips were immunostained with anti- $\gamma \mathrm{H} 2 \mathrm{AX}$ (cat. no. 05-636; EMD Millipore) antibody overnight at $4^{\circ} \mathrm{C}$. On the next day, the coverslips were washed for three times and incubated for $1 \mathrm{~h}$ using secondary antibody Alexa Fluor 488-conjugated goat anti-mouse IgG (1:50; cat. no. 4408; Cell Signaling Technology, Inc.) at room temperature. The nuclei were counterstained using DAPI (4',6-diamidino-2-phenylindole; Sangon Biotech). Olympus laser scanning confocal microscope (Olympus Optical Co., Tokyo, Japan) was used to capture images. The $\gamma \mathrm{H} 2 \mathrm{AX}$ foci were counted from at least 50 cells per sample.

Cell cycle analysis. Cells were treated with the indicated drugs, and then were collected and fixed in $70 \%$ ethanol overnight at $4^{\circ} \mathrm{C}$. On the next day, the cells were washed, suspended and stained using propidium iodide (PI) staining solution $(50 \mu \mathrm{g} / \mathrm{ml}$ PI and $1 \mathrm{mg} / \mathrm{ml}$ RNase in PBS). The cell cycle analysis was conducted using flow cytometer FACScan (Beckman Coulter, Inc.).

Invasion and migration assays: Polycarbonate membrane Transwell inserts (8- $\mu \mathrm{m}$ pore size) in a 24-well format (Corning, Inc., Corning, NY, USA) were used. For the invasion assay, the inserted membranes were coated with Matrigel (BD Biosciences, San Jose, CA, USA). For the migration assay, there was no Matrigel on the filter. The cells treated with the indicated drugs were planted into the membranes of the upper chambers in $200 \mu \mathrm{l}$ of serum-free medium at a density of $1 \times 10^{5}$ cells/chamber, which was inserted into the lower wells containing $500 \mu \mathrm{l}$ of $10 \%$ FBS-supplemented medium. Twenty-four hours later, the cells were fixed with $100 \%$ methanol and stained with $0.1 \%$ crystal violet solution. Cells on the upper side of the filter were removed with cotton swabs. Cells on the underside of the filter were counted in three randomly chosen fields and photographed using an inverted microscope (magnification, x200; Olympus Optical Co.).

Statistical analysis. Each experiment was repeated for at least three times. The data are presented as mean \pm standard deviation (SD) and were analyzed using SPSS 17.0 software (SPSS, Inc., Chicago, IL, USA). The Student's t-test or ANOVA was used. A probability level of 0.05 was defined to indicate a statistically significant difference.

\section{Results}

PI3K inhibitor BKM120 synergizes with PARP inhibitor olaparib to inhibit the proliferation of gastric cancer cells with ARIDIA deficiency. Human gastric cancer cell lines (AGS and SNU-1) were transfected with ARIDIA shRNAs (sh-AR\#1 and sh-AR\#2), and the control cells were transfected with sh-Luc. The efficacy of ARIDIA knockdown was evaluated by western blot analysis (Fig. 1A).

To explore the sensitivity of gastric cancer cells to BKM120 and olaparib, the above cells were exposed to BKM120, olaparib, or the combination at indicated concentrations. In addition, the MTS assay was conducted. The results revealed that BKM120 and olaparib inhibited the proliferation of both gastric cancer cell lines in a dose-dependent manner (Fig. 1B). The $\mathrm{IC}_{50}$ values calculated for BKM120 for the control (sh-Luc) of AGS and SNU-1 cells were higher $(1.78 \pm 0.89$ for AGS and $1.13 \pm 0.34 \mu \mathrm{mol} / 1$ for SNU-1) than those for the ARID1A-knockdown cells $(1.30 \pm 0.65$ and $1.36 \pm 0.59 \mu \mathrm{mol} / 1$ for AGS, $0.86 \pm 0.43$ and $0.78 \pm 0.32 \mu \mathrm{mol} / 1$ for SNU-1, respectively). In addition, marked synergy was observed between BKM120 and olaparib in the ARID1Adepleted cells (CI value was 0.74 and 0.70 in AGS, and 0.82 and 0.63 in SNU-1 cells), but was not observed in the control cells (CI, 0.95 in AGS and 1.05 in SNU-1).

Additionally, clonogenic assay was performed to verify the synergistic role of BKM120 and olaparib (Fig. 1C). The results demonstrated that in all AGS and SNU-1 cells, BKM120 as a single-agent markedly reduced the number of clones, as well as olaparib. Compared with olaparib, the combined treatment with BKM120 and olaparib led to significantly additional inhibition in all gastric cancer cells $(\mathrm{P}<0.05)$. Whereas, compared with BKM120, the combined treatment resulted in significantly further inhibition only in ARID1A-depleted cells, but not in the control cells (Fig. 1D), suggesting that the synergistic role of the combination was notable in gastric cancer cells with ARID1A depletion.

Combination with BKM120 and olaparib induces a higher percentage of apoptosis in ARIDIA-depleted gastric cancer cells. ARIDIA was transiently knocked down in AGS and SNU-1 cells by si-RNA ( $\mathrm{si-AR}$ ), and western blot analysis was used to evaluate the knockdown efficacy (Fig. 2A).

To identify the possible mechanisms of the synergistic effect between BKM120 and olaparib, an apoptosis assay was performed (Fig. 2B). The results demonstrated that BKM120 or olaparib induced apoptosis when used alone. However, the combined treatment with BKM120 and olaparib significantly increased apoptosis compared to that of either single-agent in the ARID1A-depleted AGS cells, but not in the control cells (si-NC) (Fig. 2C). 

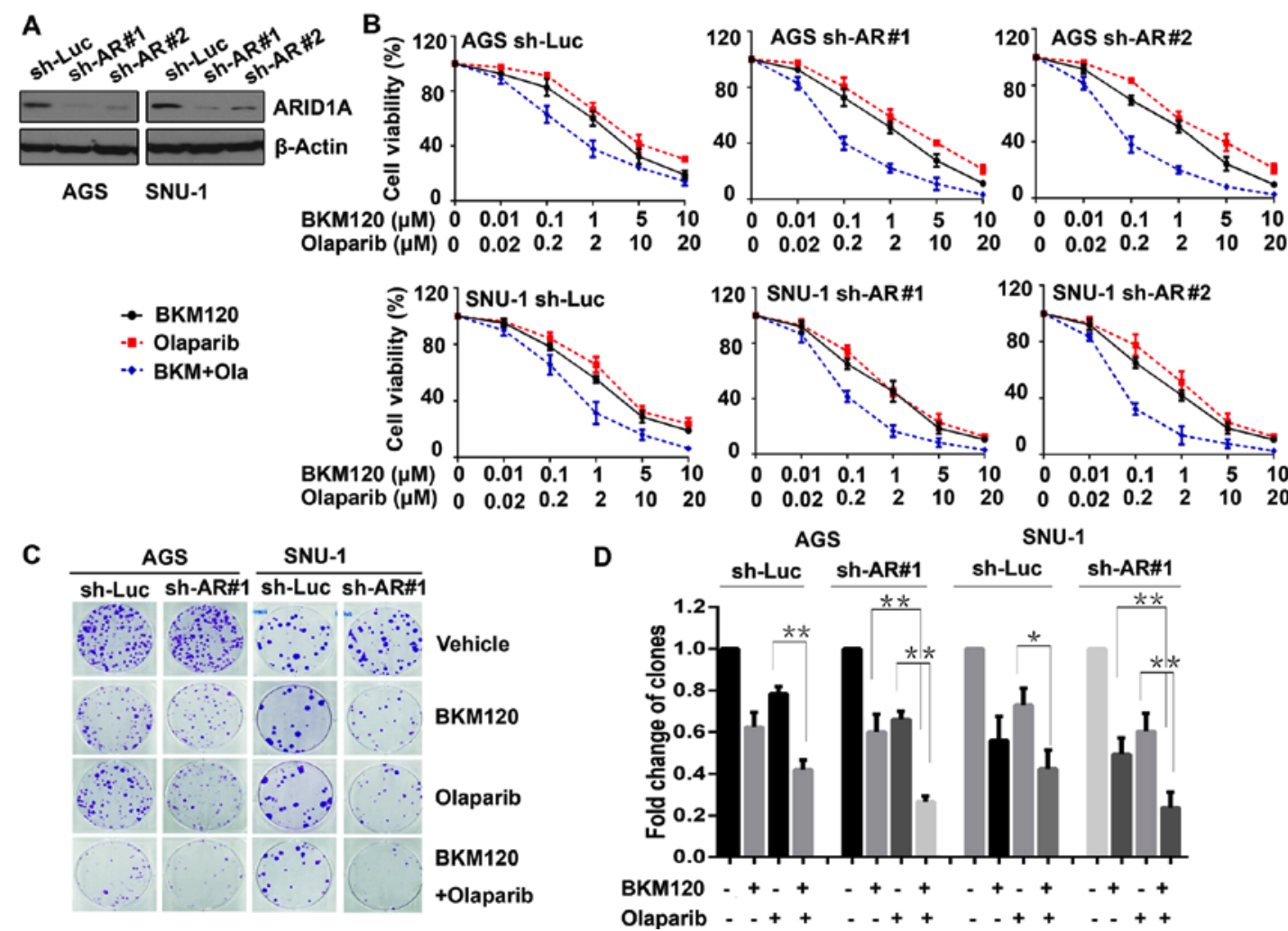

Figure 1.PI3K inhibitor BKM120 synergizes with PARP inhibitor olaparib to inhibit the proliferation of gastric cancer cells with ARID1A deficiency. (A) ARID1A was stably knocked down in AGS and SNU-1 cell lines by shARIDIA \#1 (sh-AR\#1) and shARIDIA \#2 (sh-AR\#2). Control cells were transfected using sh-Luc. Results of the western blot analysis confirmed the knockdown efficacy. (B) BKM120 synergized with olaparib to inhibit the proliferation of gastric cancer cells with ARID1A depletion. The cells were treated with the indicated concentrations of BKM120, olaparib, or the combination for $72 \mathrm{~h}$, and then MTS assay was used to measure the cell proliferation. OD value was evaluated and plotted with respect to concentration. (C) Clonogenic assays were conducted in AGS and SNU-1 cells with or without ARIDIA knockdown using DMSO (vehicle), BKM120 (1 $\mu \mathrm{mol} / 1)$, olaparib (2 $\mu$ mol/l), or BKM120 (1 $\mu$ mol/l) combined with olaparib ( $2 \mu \mathrm{mol} / 1)$. (D) Three independent experiments were performed, and the results are presented in a bar graph using the mean \pm SD. ${ }^{*} \mathrm{P}<0.05$ and ${ }^{* *} \mathrm{P}<0.01$; Luc, luciferase.

A

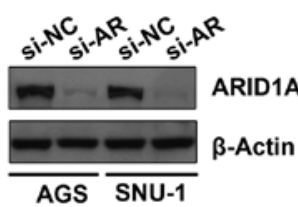

B

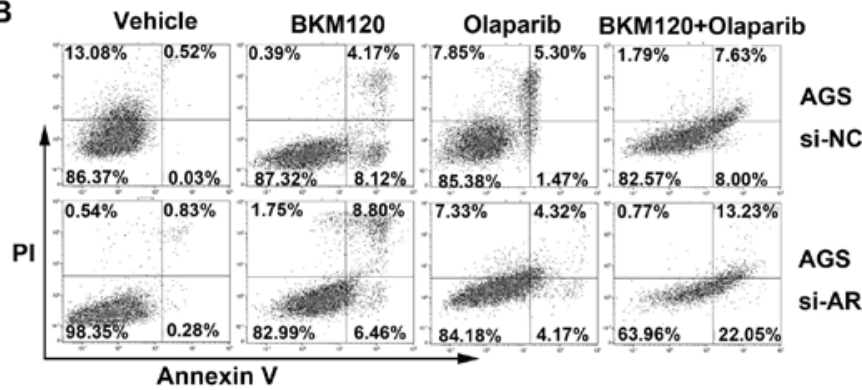

C

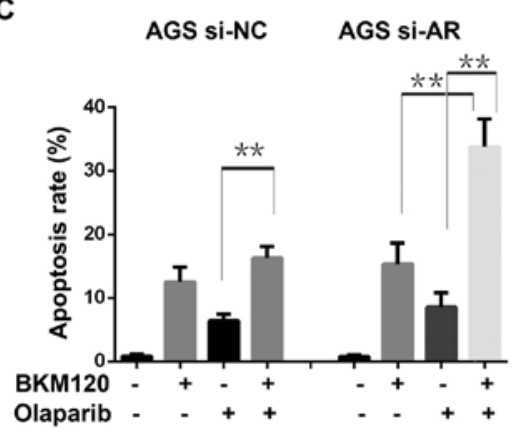

D

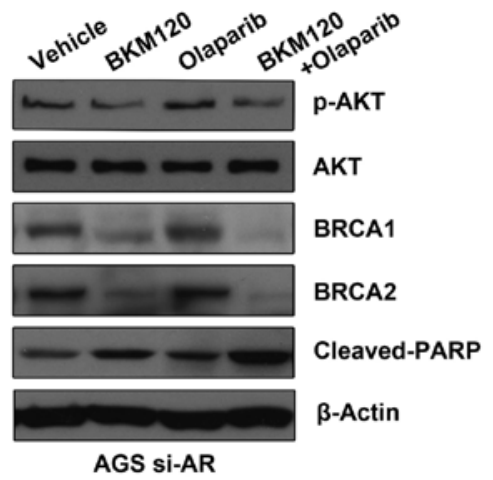

Figure 2. Combination with BKM120 and olaparib induces a higher percentage of apoptosis in ARID1A-deficient gastric cancer cells. (A) ARID1A was transiently knocked down in AGS and SNU-1 cell lines by si-AR. Control cells were treated using si-NC. Results of the western blot analysis confirmed the knockdown efficacy. (B) Apoptosis was detected by flow cytometry in ARID1A-depleted (si-AR) or control (si-NC) AGS cells treated with DMSO (vehicle), BKM120 $(1 \mu \mathrm{mol} / 1)$, olaparib $(2 \mu \mathrm{mol} / 1)$, or BKM120 $(1 \mu \mathrm{mol} / 1)$ combined with olaparib $(2 \mu \mathrm{mol} / \mathrm{l})$. (C) The bar graph shows the quantitative results representing the mean \pm SD of three independent experiments. ${ }^{* *} \mathrm{P}<0.01$. (D) Western blot analysis was used to detect protein expression in transiently ARID1A-depleted AGS cells (AGS si-AR) treated with the indicated drugs. NC, non-target control; AR, ARID1A. 
A
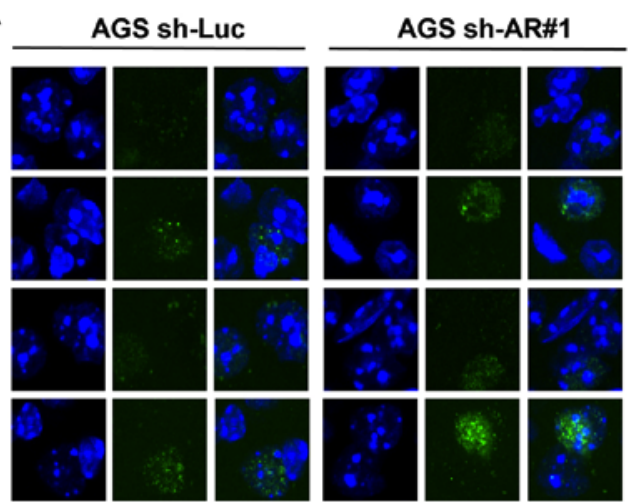

C

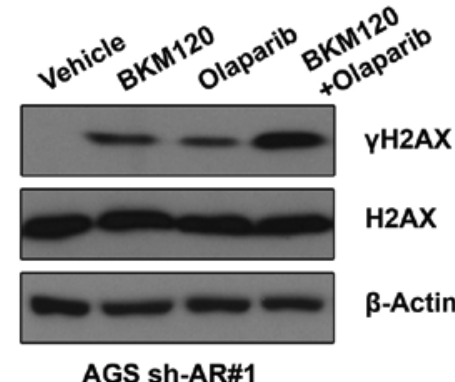

B Vehicle BKM120 Olaparib

BKM120 +Olaparib

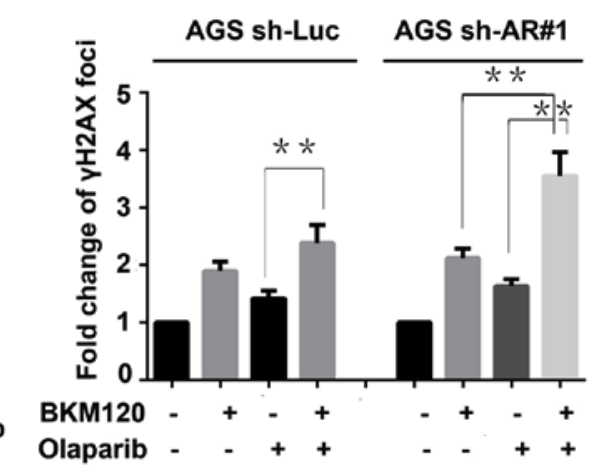

D

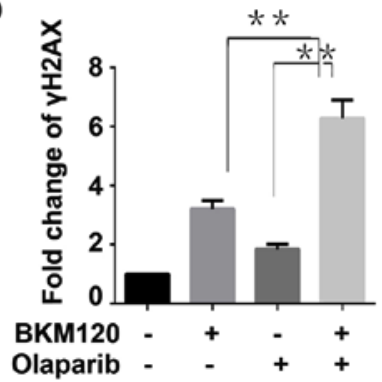

Figure 3. BKM120 combined with olaparib induces significant DNA damage in gastric cancer cells with ARID1A deficiency. (A) The immunofluorescence staining for $\gamma \mathrm{H} 2 \mathrm{AX}$ foci was performed in control (AGS sh-Luc) and ARID1A-depleted AGS (AGS sh-AR\#1) cells treated with the indicated drugs for $72 \mathrm{~h}$. (C) Western blot analysis for $\gamma \mathrm{H} 2 \mathrm{AX}$ and H2AX protein was conducted in AGS sh-AR\#1 cells treated with the indicated drugs. (B and D) The bar graphs show the quantitative results representing the mean $\pm \mathrm{SD}$ of three independent experiments. ${ }^{* *} \mathrm{P}<0.01$. Luc, luciferase; AR, ARID1A.

Furthermore, western blot analysis was applied to detect the expression level of proteins including phosphorylated AKT (p-AKT) at Ser473, AKT, BRCA1/2 and cleaved PARP in AGS cells with ARID1A deficiency (si-AR). The results revealed that BKM120 either as a single-agent or in combination with olaparib substantially reduced the abundance of p-AKT, a downstream effector of PI3K, as well as BRCA1 and BRCA2. In accordance with the results of the apoptosis assay, combined use of BKM120 and olaparib significantly enhanced the abundance of cleaved PARP, a marker of active apoptosis, compared to that of either BKM120 or olaparib (Fig. 2D).

BKM120 combined with olaparib induces significant DNA damage in gastric cancer cells with ARIDIA deficiency. To evaluate the effect of the drugs on DNA damage, the DNA damage marker $\gamma \mathrm{H} 2 \mathrm{AX}$ was detected by immunofluorescence assay (Fig 3A). The results revealed that the $\gamma \mathrm{H} 2 \mathrm{AX}$ foci were markedly increased by BKM120 or olaparib compared to that of the control (vehicle) in the AGS cells. In the control (sh-Luc) cells, the dual inhibition of PI3K and PARP significantly increased more $\gamma \mathrm{H} 2 \mathrm{AX}$ foci only when compared with the cells treated with olaparib, but not to BKM120. However, in the ARID1A-depleted (sh-AR\#1) AGS cells, the combined treatment significantly induced more $\gamma \mathrm{H} 2 \mathrm{AX}$ foci than both single BKM120 and olaparib treatments $(\mathrm{P}<0.01)$ (Fig. 3B). These results suggested that the synergistic role of BKM120 and olaparib in inducing DNA damage was more remarkable in ARID1A-deficient gastric cancer cells.

Subsequently, western blot analysis was performed to detect the abundance of $\gamma \mathrm{H} 2 \mathrm{AX}$ in ARID1A-depleted AGS cells treated with the indicated drugs (Fig. 3C). The results indi- cated that the combined treatment with BKM120 and olaparib increased the abundance of $\gamma \mathrm{H} 2 \mathrm{AX}$ more significantly than both the single-agent groups and the control group (vehicle), in the ARID1A-knockdown AGS cells (AGS sh-AR\#1) (Fig. 3D).

G2-M checkpoint arrest induced by the combined treatment with BKM120 and olaparib is significantly attenuated in ARID1A-deficient gastric cancer cells. Since G2-M checkpoint arrest is critical to DNA damage repair, the cell cycle distribution was detected by FACS analysis in AGS cells with or without ARID1A depletion (Fig. 4A). The results indicated that single BKM120 or olaparib activated G2-M phase arrest compared with the vehicle group, which was a response to the increased DNA damage that they induced. The combination of BKM120 and olaparib enhanced the G2-M phase arrest more significantly than single BKM120 or olaparib. However, in the ARID1A-depleted AGS cells (AGS si-AR), the G2-M phase arrest induced by the combination was weaker compared to that of the control (si-NC) cells (Fig. 4B). The cell cycle analysis in SNU-1 cells revealed similar results (Fig. 4C and D), suggesting that ARID1A depletion attenuated the G2-M checkpoint arrest induced by the combined treatment.

BKM120 combined with olaparib inhibits migration and invasion of gastric cancer cells with ARIDIA depletion. Invasion and migration are crucial to the growth and metastasis of cancer. Thus, invasion and migration assays were performed using ARID1A-depleted AGS (AGS sh-AR\#1) cells treated with vehicle, BKM120, olaparib or the combination (Fig. 5A). The results revealed that both BKM120 and olaparib inhibited the migration and invasion of the gastric cancer cells, 

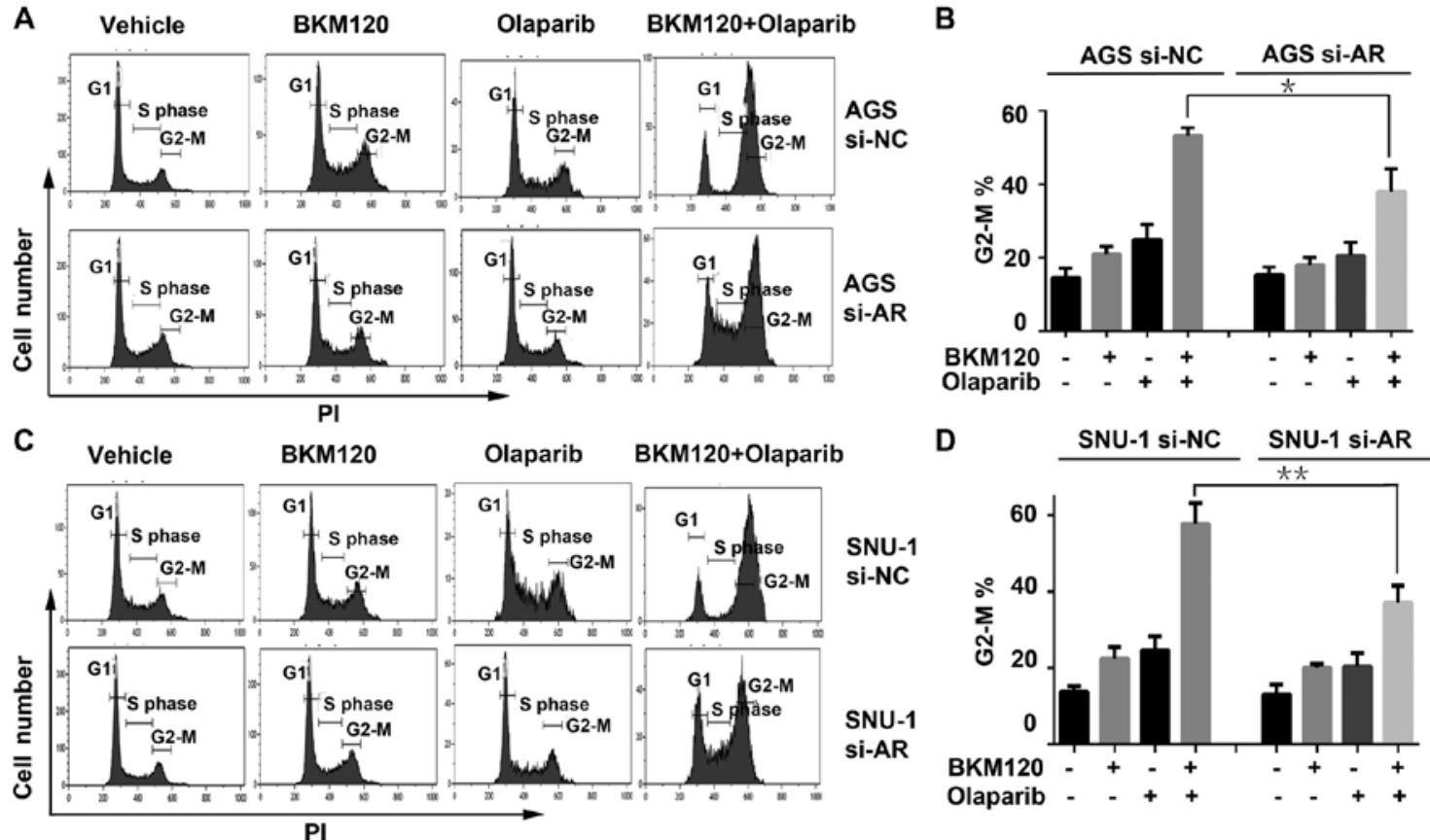

Figure 4. G2-M checkpoint arrest induced by the combined treatment with BKM120 and olaparib is significantly attenuated in ARID1A-deficient gastric cancer cells. (A and C) The cell cycle analysis was conducted in control (si-NC) and ARID1A-silencing (si-AR) AGS or SNU-1 cells treated with the indicated drugs. (B and D) The bar graphs show the quantitative results representing the mean $\pm \mathrm{SD}$ of three independent experiments. ${ }^{*} \mathrm{P}<0.05,{ }^{* * *} \mathrm{P}<0.01 ; \mathrm{AR}, \mathrm{ARID} 1 \mathrm{~A}$; NC, non-target control; PI, propidium iodide.

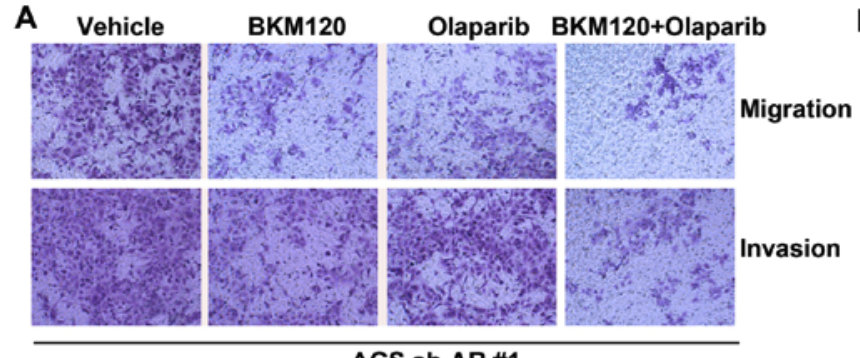

AGS sh-AR \#1

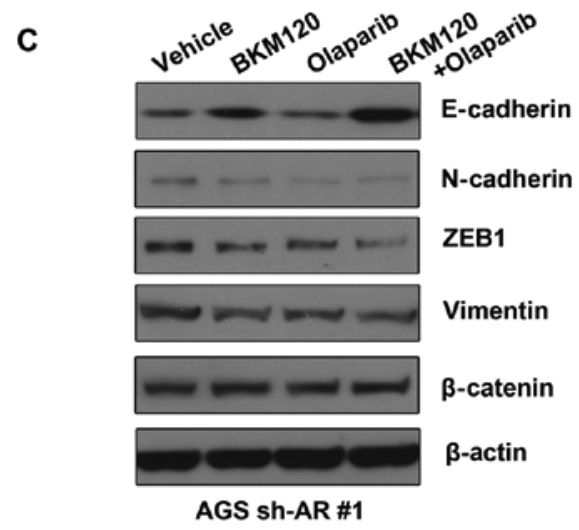

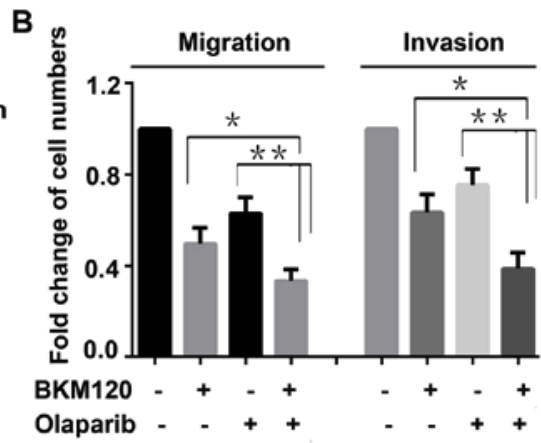

D

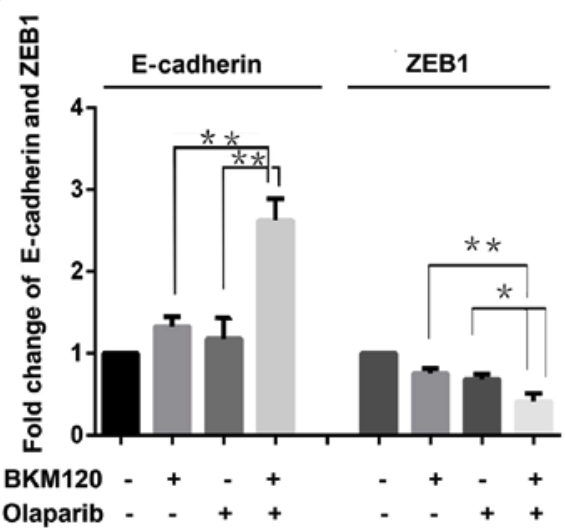

Figure 5. BKM120 combined with olaparib inhibits the migration and invasion of gastric cancer cells with ARID1A deficiency. (A) The migration and invasion assays were performed in ARID1A-depleted AGS (AGS sh-AR\#1) treated with the indicated drugs. (C) Western blot analysis was used to detect the abundance of migration-related proteins in AGS cells with ARID1A deficiency treated using the indicated drugs. (B and D) The bar graphs showed the quantitative results representing the mean $\pm \mathrm{SD}$ of three independent experiments. ${ }^{*} \mathrm{P}<0.05,{ }^{* *} \mathrm{P}<0.01 ; \mathrm{AR}, \mathrm{ARID} 1 \mathrm{~A}$.

compared to that of the control group. However, the dual treatment with BKM120 and olaparib resulted in the lowest degree of migration and invasion, compared to that of either BKM120 or olaparib as a single-agent treatment (Fig. 5B). 
Moreover, western blot analysis was conducted to evaluate the abundance of proteins involving in invasion and migration, including $\mathrm{N}$-cadherin, E-cadherin, $\beta$-catenin, ZEB1 and vimentin in the ARID1A-depleted AGS (AGS sh-AR\#1) cells (Fig. 5C). The results showed that compared to that of the control (vehicle) group, the abundance of E-cadherin increased, while N-cadherin, ZEB1 and vimentin decreased in both the BKM120 and olaparib groups. In the group treated with the combination of BKM120 and olaparib, the increase in E-vadherin and the decrease in ZEB1 were more significant than both of the single-agent treatment groups (Fig. 5D).

\section{Discussion}

ARID1A has been demonstrated to regulate gastric cancer cell proliferation and migration $(22,34,36)$. ARID1A-deficient cancer cells are reported to be sensitive to inhibition of PI3K and PARP. In the present study, we revealed that the combination of PI3K inhibitor BKM120 and PARP inhibitor olaparib had synergistic antitumor effects on gastric cancer cells with ARID1A deficiency, via inhibiting cell proliferation, inducing apoptosis, increasing DNA damage, attenuating G2-M checkpoint arrest and suppressing invasion and migration. To the best of our knowledge, this is the first study to explore the effect of the combination of BKM120 and olaparib on gastric cancer, suggesting ARID1A deficiency as a potential predictive biomarker.

Recently, Zhang et al reported that ARID1A deficiency increased the transcription of PI3K, and activated the PI3K/AKT pathway in gastric cancer. Consequently, PI3Kinhibitor LY294002 and AKT-inhibitor mk2206 were highly effective on gastric cancer cells harboring deficient ARID1A in vitro and in vivo (22). Similarly, several other studies also revealed that the loss of ARID1A expression upregulated the phosphorylation of AKT, which was a component of the PI3K signaling pathway, in endometrial cancer (37), ovarian clear cell carcinoma (38), and colon cancer (39). The synthetic lethal interaction between loss of ARID1A expression and inhibition of the PI3K/AKT pathway was also reported by Samartzis et al in breast and ovarian cancer cells (40). Meanwhile, Shen et al reported that ARID1A deficiency impaired the DNA damage checkpoint and sensitized cells to PARP inhibitors (12). In accordance with the previous findings, herein, we demonstrated that BKM120 and olaparib significantly suppressed the proliferation of gastric cancer cells with ARID1A depletion.

PARP inhibitors show a synergistic effect with several therapeutic reagents, such as EGFR inhibitors (41) and PI3K/ mTOR inhibitors (42). The combination with olaparib and BKM120 has been reported to be synergistically effective in breast $(14,18)$, prostate (19), and ovarian cancer with or without PIK3CA mutations $(20,21)$. In the present study, the results demonstrated that olaparib synergized with BKM120 to effectively inhibit the proliferation of gastric cancer cells harboring ARID1A deficiency. Mechanistically, BKM120 downregulated BRCA1/2 expression, which mimicked the mutations of BRCA1/2, and might sensitize cells to PARP inhibitors.

BKM120 has been reported to induce DNA damage in breast (14,18), glioblastoma (43), ovarian and prostate cancer (19). We revealed that either BKM120 or olaparib, when used as a single-agent treatment, induced DNA damage. Nonetheless, the combined treatment with BKM120 and olaparib potentiated the DNA damage, especially in ARID1A-deficient gastric cancer cells, which may result in the synergistic anticancer effect of BKM120 and olaparib.

Normally, after DNA damage, the cell cycle checkpoint is activated, which leads to arrest injury, repair the DNA damage and protect cells from apoptosis and unscheduled death (44). The previous studies and our results revealed that PI3K inhibitor and PARP inhibitor activated the G2-M checkpoint, and caused cell cycle arrest, until the DNA damage was repaired $(44,45)$. We also revealed that the combination with BKM120 and olaparib synergistically enhanced G2-M phase arrest. However, the G2-M checkpoint arrest induced by the combined treatment was significantly attenuated in ARID1Adeficient gastric cancer cells, although the DNA damage in these cells was the most serious. It has been reported that ARID1A is required for a proper G2-M DNA damage checkpoint, and ARID1A deficiency impairs the G2-M arrest and DNA damage repair (12). Due to usual deficient function in G1-M checkpoint, most cancer cells depend on the G2-M cell cycle checkpoint (44). Therefore, we propose that ARID1A deficiency attenuates the G2-M checkpoint arrest induced by the combined treatment, which may weaken the DNA damage repair, and eventually lead to genomic instability, cell apoptosis and death. Accordingly, another study in gastric cancer has reported that ARID1A mutations are associated with increased microsatellite instability (MSI), which is a form of genomic instability (26).

Invasion and migration are crucial to tumor growth and metastasis. In addition, reduced expression of ARID1A has been reported to enhance the migration and invasion of gastric cancer cells via downregulation of E-cadherin transcription (34). Thus, for the drugs intended to treat gastric cancer harboring ARID1A deficiency, it is important to gain knowledge concerning the efficacy of these drugs on migration and invasion. In addition, Wang et al reported that dual treatment with BKM120 and olaparib significantly inhibited the invasion and migration of ovarian cancer cells (20). Previous studies have reported that ZEB1 plays an important role in suppressing E-cadherin expression and in promoting tumor invasion and metastasis $(46,47)$. In accordance with these findings, our results revealed that the dual blockade of PI3K and PARP notably suppressed the invasion and migration in gastric cancer cells with ARID1A depletion through a decrease in ZEB1 and an increase in E-cadherin, which is critical for the utilization of such a drug combination in vivo and in the clinic therapeutically.

In summary, we demonstrated that the PI3K inhibitor BKM120 synergized with the PARP inhibitor olaparib to inhibit the growth and migration of gastric cancer cells with ARID1A deficiency in vitro. The present study provides a potential therapeutic strategy for gastric cancer harboring ARID1A deficiency, which warrants verification in vivo and in the clinic in future research.

\section{Acknowledgements}

We would like to thank Dr Hai Zhang (Department of Physiology and Research, Tongji Medical College, Huazhong University of Science and Technology) for his contributions to the immunofluorescent staining. 


\section{Funding}

The present study was supported by the Natural Science Foundation of Hubei Province (no. 2015CFB541) and the Research Project of Hubei provincial health and Family Planning Commission (no. WJ2017M114).

\section{Availability of data and materials}

The datasets used in the present study are available from the corresponding author upon reasonable request.

\section{Authors' contributions}

GH, LY and YD designed and supervised the project. LY, GY and YH performed the experiments. SL and LZ analyzed the data and prepared the graphs. LY, WW and JW wrote the manuscript. GH and YD edited the manuscript. All authors read and approved the manuscript and agree to be accountable for all aspects of the research in ensuring that the accuracy or integrity of any part of the work are appropriately investigated and resolved.

\section{Ethics approval and consent to participate}

No animal or human experiments were included in the present study.

\section{Consent for publication}

Not applicable.

\section{Competing interests}

The authors state that they have no competing interests.

\section{References}

1. Venerito M, Link A, Rokkas T and Malfertheiner P: Gastric cancer - clinical and epidemiological aspects. Helicobacter 21 (Suppl 1): S39-S44, 2016.

2. Torre LA, Bray F, Siegel RL, Ferlay J, Lortet-Tieulent J and Jemal A: Global cancer statistics, 2012. CA Cancer J Clin 65 87-108, 2015

3. Fitzmaurice C, Dicker D, Pain A, Hamavid H, Moradi-Lakeh M, MacIntyre MF, Allen C, Hansen G, Woodbrook R, Wolfe C, et al; Global Burden of Disease Cancer Collaboration: The Global Burden of Cancer 2013. JAMA Oncol 1: 505-527, 2015.

4. Ferlay J, Shin HR, Bray F, Forman D, Mathers C and Parkin DM: Estimates of worldwide burden of cancer in 2008: GLOBOCAN 2008. Int J Cancer 127: 2893-2917, 2010.

5. Ajani JA, D'Amico TA, Almhanna K, Bentrem DJ, Chao J, Das P, Denlinger CS, Fanta P, Farjah F, Fuchs CS, et al: Gastric Cancer, Version 3.2016, NCCN Clinical Practice Guidelines in Oncology. J Natl Compr Canc Netw 14: 1286-1312, 2016.

6. Deeks ED: Olaparib: First global approval. Drugs 75: 231-240, 2015.

7. Farmer H, McCabe N, Lord CJ, Tutt AN, Johnson DA, Richardson TB, Santarosa M, Dillon KJ, Hickson I, Knights C, et al: Targeting the DNA repair defect in BRCA mutant cells as a therapeutic strategy. Nature 434: 917-921, 2005.

8. Bryant HE, Schultz N, Thomas HD, Parker KM, Flower D, Lopez E, Kyle S, Meuth M, Curtin NJ and Helleday T: Specific killing of BRCA2-deficient tumours with inhibitors of poly(ADP-ribose) polymerase. Nature 434: 913-917, 2005.
9. Alagpulinsa DA, Ayyadevara S, Yaccoby S and Shmookler Reis RJ: A cyclin-dependent kinase inhibitor, dinaciclib, impairs homologous recombination and sensitizes multiple myeloma cells to PARP inhibition. Mol Cancer Ther 15: 241-250, 2016

10. Min A, Im SA, Yoon YK, Song SH, Nam HJ, Hur HS, Kim HP, Lee KH, Han SW, Oh DY, et al: RAD51C-deficient cancer cells are highly sensitive to the PARP inhibitor olaparib. Mol Cancer Ther 12: 865-877, 2013.

11. Kubota E, Williamson CT, Ye R, Elegbede A, Peterson L, Lees-Miller SP and Bebb DG: Low ATM protein expression and depletion of p53 correlates with olaparib sensitivity in gastric cancer cell lines. Cell Cycle 13: 2129-2137, 2014.

12. Shen J, Peng Y, Wei L, Zhang W, Yang L, Lan L, Kapoor P, Ju Z, Mo Q, Shih IeM, et al: ARID1A deficiency impairs the DNA damage checkpoint and sensitizes cells to PARP inhibitors. Cancer Discov 5: 752-767, 2015.

13. Bang YJ, Xu RH, Chin K, Lee KW, Park SH, Rha SY, Shen L, Qin S, Xu N, Im SA, et al: Olaparib in combination with paclitaxel in patients with advanced gastric cancer who have progressed following first-line therapy (GOLD): A double-blind, randomised, placebo-controlled, phase 3 trial. Lancet Oncol 18: 1637-1651, 2017.

14. Ibrahim YH, García-García C, Serra V, He L, Torres-Lockhart K, Prat A, Anton P, Cozar P, Guzmán M, Grueso J, et al: PI3K inhibition impairs BRCA1/2 expression and sensitizes BRCAproficient triple-negative breast cancer to PARP inhibition. Cancer Discov 2: 1036-1047, 2012.

15. Engelman JA: Targeting PI3K signalling in cancer: Opportunities, challenges and limitations. Nat Rev Cancer 9: 550-562, 2009

16. Cantley LC: The phosphoinositide 3-kinase pathway. Science 296: 1655-1657, 2002.

17. Park E, Park J, Han SW, Im SA, Kim TY, Oh DY and Bang YJ: NVP-BKM120, a novel PI3K inhibitor, shows synergism with a STAT3 inhibitor in human gastric cancer cells harboring KRAS mutations. Int J Oncol 40: 1259-1266, 2012.

18. Juvekar A, Burga LN, Hu H, Lunsford EP, Ibrahim YH, Balmañà J, Rajendran A, Papa A, Spencer K, Lyssiotis CA, et al: Combining a PI3K inhibitor with a PARP inhibitor provides an effective therapy for BRCA1-related breast cancer. Cancer Discov 2: 1048-1063, 2012.

19. González-Billalabeitia E, Seitzer N, Song SJ, Song MS Patnaik A, Liu XS, Epping MT, Papa A, Hobbs RM, Chen M, et al: Vulnerabilities of PTEN-TP53-deficient prostate cancers to compound PARP-PI3K inhibition. Cancer Discov 4: 896-904, 2014.

20. Wang D, Wang M, Jiang N, Zhang Y, Bian X, Wang X, Roberts TM, Zhao JJ, Liu P and Cheng H: Effective use of PI3K inhibitor BKM120 and PARP inhibitor Olaparib to treat PIK3CA mutant ovarian cancer. Oncotarget 7: 13153-13166, 2016.

21. Wang D, Li C, Zhang Y, Wang M, Jiang N, Xiang L, Li T, Roberts TM, Zhao JJ, Cheng H, et al: Combined inhibition of PI3K and PARP is effective in the treatment of ovarian cancer cells with wild-type PIK3CA genes. Gynecol Oncol 142: 548-556, 2016.

22. Zhang Q, Yan HB, Wang J, Cui SJ, Wang XQ, Jiang YH, Feng L, Yang PY and Liu F: Chromatin remodeling gene AT-rich interactive domain-containing protein 1A suppresses gastric cancer cell proliferation by targeting $P I K 3 C A$ and $P D K 1$. Oncotarget 7: 46127-46141, 2016.

23. Jones S, Wang TL, Shih IeM, Mao TL, Nakayama K, Roden R, Glas R, Slamon D, Diaz LA Jr, Vogelstein B, et al: Frequent mutations of chromatin remodeling gene ARIDIA in ovarian clear cell carcinoma. Science 330: 228-231, 2010.

24. Kandoth C, Schultz N, Cherniack AD, Akbani R, Liu Y, Shen H, Robertson AG, Pashtan I, Shen R, Benz CC, et al; Cancer Genome Atlas Research Network: Integrated genomic characterization of endometrial carcinoma. Nature 497: 67-73, 2013.

25. Takeda T, Banno K, Okawa R, Yanokura M, Iijima M, Irie-Kunitomi H, Nakamura K, Iida M, Adachi M, Umene K, et al: ARIDIA gene mutation in ovarian and endometrial cancers (Review). Oncol Rep 35: 607-613, 2016.

26. Wang K, Kan J, Yuen ST, Shi ST, Chu KM, Law S, Chan TL, Kan Z, Chan AS, Tsui WY, et al: Exome sequencing identifies frequent mutation of ARIDIA in molecular subtypes of gastric cancer. Nat Genet 43: 1219-1223, 2011.

27. Zang ZJ, Cutcutache I, Poon SL, Zhang SL, McPherson JR, Tao J, Rajasegaran V, Heng HL, Deng N, Gan A, et al: Exome sequencing of gastric adenocarcinoma identifies recurrent somatic mutations in cell adhesion and chromatin remodeling genes. Nat Genet 44: 570-574, 2012 
28. Ali SM, Sanford EM, Klempner SJ, Rubinson DA, Wang K, Palma NA, Chmielecki J, Yelensky R, Palmer GA, Morosini D, et al: Prospective comprehensive genomic profiling of advanced gastric carcinoma cases reveals frequent clinically relevant genomic alterations and new routes for targeted therapies. Oncologist 20: 499-507, 2015.

29. Roberts CW and Orkin SH: The SWI/SNF complex - chromatin and cancer. Nat Rev Cancer 4: 133-142, 2004.

30. Mao TL and Shih IeM: The roles of ARID1A in gynecologic cancer. J Gynecol Oncol 24: 376-381, 2013.

31. Nagl NG Jr, Patsialou A, Haines DS, Dallas PB, Beck GR Jr and Moran E: The p270 (ARID1A/SMARCF1) subunit of mammalian SWI/SNF-related complexes is essential for normal cell cycle arrest. Cancer Res 65: 9236-9244, 2005.

32. Wu JN and Roberts CW: ARID1A mutations in cancer: Another epigenetic tumor suppressor? Cancer Discov 3: 35-43, 2013.

33. Guan B, Wang TL and Shih IeM: ARIDIA, a factor that promotes formation of SWI/SNF-mediated chromatin remodeling, is a tumor suppressor in gynecologic cancers. Cancer Res 71 6718-6727, 2011

34. Yan HB, Wang XF, Zhang Q, Tang ZQ, Jiang YH, Fan HZ, Sun YH, Yang PY and Liu F: Reduced expression of the chromatin remodeling gene ARIDIA enhances gastric cancer cell migration and invasion via downregulation of E-cadherin transcription. Carcinogenesis 35: 867-876, 2014.

35. Chou TC: Drug combination studies and their synergy quantification using the Chou-Talalay method. Cancer Res 70: 440-446, 2010.

36. Wang DD, Chen YB, Pan K, Wang W, Chen SP, Chen JG, Zhao JJ, Lv L, Pan QZ, Li YQ, et al: Decreased expression of the ARIDIA gene is associated with poor prognosis in primary gastric cancer. PLoS One 7: e40364, 2012.

37. Liang H, Cheung LW, Li J, Ju Z, Yu S, Stemke-Hale K, Dogruluk T, Lu Y, Liu X, Gu C, et al: Whole-exome sequencing combined with functional genomics reveals novel candidate driver cancer genes in endometrial cancer. Genome Res 22: 2120-2129, 2012
38. Chandler RL, Damrauer JS, Raab JR, Schisler JC, Wilkerson MD, Didion JP, Starmer J, Serber D, Yee D, Xiong J, et al: Coexistent ARID1A-PIK3CA mutations promote ovarian clear-cell tumorigenesis through pro-tumorigenic inflammatory cytokine signalling. Nat Commun 6: 6118, 2015.

39. Xie C, Fu L, Han Y, Li Q and Wang E: Decreased ARID1A expression facilitates cell proliferation and inhibits 5-fluorouracil-induced apoptosis in colorectal carcinoma. Tumour Biol 35: 7921-7927, 2014

40. Samartzis EP, Gutsche K, Dedes KJ, Fink D, Stucki M and Imesch P: Loss of ARID1A expression sensitizes cancer cells to PI3K- and AKT-inhibition. Oncotarget 5: 5295-5303, 2014.

41. Nowsheen S, Cooper T, Stanley JA and Yang ES: Synthetic lethal interactions between EGFR and PARP inhibition in human triple negative breast cancer cells. PLoS One 7: e46614, 2012.

42. Cardnell RJ, Feng Y, Mukherjee S, Diao L, Tong P, Stewart CA, Masrorpour F, Fan Y, Nilsson M, Shen Y, et al: Activation of the $\mathrm{PI} 3 \mathrm{~K} / \mathrm{mTOR}$ pathway following PARP inhibition in small cell lung cancer. PLoS One 11: e0152584, 2016.

43. Jane EP, Premkumar DR, Morales A, Foster KA and Pollack IF. Inhibition of phosphatidylinositol 3-kinase/AKT signaling by NVP-BKM120 promotes ABT-737-induced toxicity in a caspase-dependent manner through mitochondrial dysfunction and DNA damage response in established and primary cultured glioblastoma cells. J Pharmacol Exp Ther 350: 22-35, 2014.

44. Yin Y, Shen Q, Zhang P, Tao R, Chang W, Li R, Xie G, Liu W, Zhang L, Kapoor P, et al: Chk1 inhibition potentiates the therapeutic efficacy of PARP inhibitor BMN673 in gastric cancer. Am J Cancer Res 7: 473-483, 2017.

45. Jiang ZB, Huang J, Xie C, Li X, Liu L, He J, Pan H, Huang L, Fan XX, Yao XJ, et al: Combined use of PI3K and MEK inhibitors synergistically inhibits lung cancer with EGFR and KRAS mutations. Oncol Rep 36: 365-375, 2016.

46. Sánchez-Tilló E, de Barrios O, Siles L, Cuatrecasas M, Castells A and Postigo A: $\beta$-catenin/TCF4 complex induces the epithelial-tomesenchymal transition (EMT)-activator ZEB1 to regulate tumor invasiveness. Proc Natl Acad Sci USA 108: 19204-19209, 2011.

47. Schmalhofer O, Brabletz S and Brabletz T: E-cadherin, betacatenin, and ZEB1 in malignant progression of cancer. Cancer Metastasis Rev 28: 151-166, 2009. 